\title{
ABSTRACTS OF CONTRIBUTED PAPERS
}

\author{
THE 2003 OUTBURST OF V4641 SGR \\ (=SAX 1819.3-2525) \\ Charles Bailyn ${ }^{1}$
}

V4641 Sgr (=SAX 1819.3-2525) is a transient black hole binary system, with a B-type secondary star. In August 2003 a new outburst of this source began. Violent variability (factors of 2 on timescales of 10 seconds or less) was seen in all wavelength regimes (radio, infrared, optical and X-ray). I will present data from our RXTE observations and OIR data from the SMARTS $1.3 \mathrm{~m}$ telescope at CTIO (ATEL $171,172)$. Observations continue as of this writing (August 8, 2003).

1 Yale University, USA

It is proposed to review recent XMM and optical (CFHT/ SUBARU) observations of ultraluminous $\mathrm{X}$-ray sources in nearby galaxies, including the apparently brightest ULXs which are located in the M81 group dwarf galaxies Holmberg II and IX. We show that their $0.2-10 \mathrm{keV}$ spectra can be characterised by simple power-law models and by negligible contribution of any (disk) thermal component, if the low-metallicity of the interstellar absorber is taken into account. Optical observations have revealed the presence of large ionized nebulae around many ULX which provide important informations on intrinsic luminosities, and on the formation and evolution of these sources. Finally, the first detection of a stellar emission line in the spectrum of an optical counterpart will be presented, and the prospects to determine the mass of an ULX will be discussed.

\footnotetext{
1 Observatcire de Strasbourg, France
}

\author{
JET INDUCED NUCLEOSYNTHESIS IN \\ MISALIGNED MICROQUASARS \\ Yousaf Butt ${ }^{1}$
}

The jet axes and the orbital planes of microquasar systems are usually assumed to be approximately perpendicular, eventhough this is not currently an observational requirement. On the contrary, in one of the few systems where the relative orientations are well-constrained, V4641Sgr, the jet axis is known to lie not more than 36 degrees from the binary plane. Such a jet, lying close to the binary plane, and traveling at a significant fraction of the speed of light may periodically impact the secondary star initiating nuclear reactions on its surface. The integrated yield of such nuclear reactions over the age of the binary system (less the radiative mass loss) will detectably alter the elemental abundances of the companion star. This scenario may explain the anomalously high $\mathrm{Li}$ enhancements (roughly 20-200 times the sun's photospheric value; or, equivalently, 0.1-1 times the average solar system value) seen in the companions of some black-hole X-ray binary systems. Gamma-ray line signatures of the proposed process could include the $2.22 \mathrm{MeV}$ neutron capture line as well as the $0.478 \mathrm{MeV} 7 \mathrm{Li}^{*}$ de-excitation line, both of which may be discernable with the INTEGRAL satellite if produced in an optically thin region during a large, close-by outburst. For very energetic jets, a relatively narrow neutral pion gamma-decay signature at 67.5 MeV could also be measurable with the GLAST satellite. We argue that about $10-20 \%$ of all microquasar systems ought to be sufficiently misaligned as to be undergoing the proposed jet-secondary impacts.

$\overline{1 \text { CfA, USA }}$ 


\section{THEORETICAL MODELS OF X-RAY BINARIES IN NEARBY GALAXIES \\ Vicky Kalogera ${ }^{1}$}

In recent years Chandra has revolutionized the study of X-ray binaries with detection of significant samples in a wide range of galaxies in the nearby Universe. Most often these populations are characterized by X-ray luminosity functions which exhibit systematic behaviors correlated with galaxian properties. We have recently developed theoretical models of X-ray binary formation and evolution that allow for the first time the direct comparison with $\mathrm{X}$ ray observations. Our first results related to starfurming galaxics ar" very encouraging and clearly show that they can confirm population properties (for example ages) derived based on observations in other wavelengths and they allow to derive physical information about $\mathrm{X}$-ray binaries in diverse environmonts.

1 Northwestern University, USA

\section{THE EXTRAORDINARY LUMINOUS BINARY STAR, QU CAR \\ Louise Hartley'}

In recent $U V$ and optical studies the interacting binary star, QU Car, has been shown to be extremely bright and highly ionised - a unique system amongst CVs. Assigned to the nova-like subclass of CVs it is, however, an order of magnitude more luminous than is typical. Furthermore, it displays spectral characteristics that would suggest a close similarity to the supersoft X-ray soure's, although it is several orders of magnitude too faint in the soft $\mathrm{X}$-ray range to be a supersoft. It has also shown to be the first known CV with a carbon-enriched companion. Here we present spectroscopic olsservations of QU Car, from the far ultraviolet through to the optical range. We show how we have built up a picture of QU Car. including its distance; it cxtremely high matis' accretion rate; and its unusual ionisation and abundance patterns. We then discuss QU Car's relation to other luminous interacting binaries, including the supersoft sources and V Sge systems, and highlight the rewards that are to be gained from understanding the evolutionary path of this system.

\footnotetext{
1 Monash University, USi
}

\section{GALACTIC POPULATIONS OF ULTRACOMPAC BINARIES Krzysztof Belczyński ${ }^{1}$}

Using the StarTrack population synthesis code we analyze the formation and evolution of ultracompact binaries (UCBs). We study UCBs formed only in the Galactic field and focus on systems with a neutron star or a black hole accretor. We predict the relative numbers of diffrent types of UCBs populating our Galaxy. We find an excellent agreement with observed sample of Galactic UCBs. Our results demonstrate that regular binary star evolution may lead to formation of all UCBs in the Galactic field. No dynamical processes of the dense stellar environments (e.g. globular clusters) are necessary to explain formation of the field UCBs. We predict that with a discovery of several more X-ray active UCBs hosting neutron stars, we should find the system with a black hole accretor.

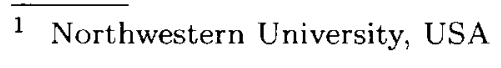

\section{TYPE IA SUPERNOVAE AND THEIR IMPLICATIONS FOR COSMOLOGY Mario Livio ${ }^{1}$}

Models for Type Ia Supernovae (SNe Ia) are reviewed. It is shown that there are strong reasons to believe that most SNe Ia represent thermonuclear disruptions of $\mathrm{C}-\mathrm{O}$ white dwarfs, when these white dwarfs reach the Chandrasekhar limit and ignite carbon at their centers.

Different progenitor scenarios are reviewed critically and the strengths and weaknesses of each scenario are presented in detail. It is argued that theoretical considerations currently favor singledegenerate models, in which the white dwarf accretes from a subgiant or giant observed sample. The recent observations of SN 2002ic and their implications are reviewed. The relation of the different models to the use of SNe Ia for the determination of cosmological parameters is discussed. It is shown that while the observed diversity of SNe Ia may argue for the existence of different progenitor classes, this does not affect the interpretation of an accelerating expansion of the universe.

Crucial observations tests of the conclusions are suggested.

\footnotetext{
$\overline{1}$ Space Telescope Science Institute, USA
} 


\section{RELATIVISTIC MEASUREMENTS OF PULSAR-WHITE DWARF BINARIES: NEW RESULTS FROM ARECIBO D. Nice ${ }^{1}$}

High precision timing observations of binary radio pulsars in nearly circular orbits with white dwarf companions have lead to new measurements and constrains on orbital geometries and component star masses. These constraints come primarily from measurements of relativistic phenomena, including precession, Shapiro delay, and orbital decay due to emission of gravitational radiation. Masses of white dwarf secondaries in these systems show good agreement with expectations from the theoretical orbital period-core mass relation. Masses of neutron star primaries in these systems show a trend of values greater than the canonical 1.35 solar masses, presumably due to mass accretion during the spin-up of the pulsars.

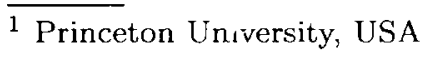

\section{MULTIWAVELENGTH OBSERVATIONS OF RX J0806.3+1527: THE SHORTEST ORBITAL PERIOD BIN \\ G. Israel ${ }^{1}$}

I will report on the results obtained from observations carried out with XMM-Newton, the Very Large telescope (VLT) and the Telescopio Nazionale
Galileo (TNG) covering the X-ray, EUV, optical and IR bands. These observations allowed us to infer new important results related to the stability of the absolute phase shift between Optical/IR and X-ray emission, the X-ray spectrum as a function of the orbital phase, the period derivative, the polarisation, and the EUV emission. These results will be discussed on the light of the theoretical models proposed so far for for RXJ0806.3+1527 and its twin source RXJ1914.4+2456

$\overline{1 \mathrm{INAF}}$ - OAR, Italy

The results of Chandra and XMM-Newton X-ray observations of V4743 Sgr (Nova Sgr 2002 no.2) carried out on several epochs after outburst will be presented here. Six months after outburst the nova had evolved into a Super-Soft X-ray source exhibiting a continuous spectrum with deep absorption features. The $\mathrm{X}$-ray light curve showed large amplitude oscillations with a period of $1325 \mathrm{sec}$ followed by a deep decline after about $13 \mathrm{ksec}$ of observations. The count rate dropped from $40 \mathrm{cts} / \mathrm{sec}$ to practically zero with 6 ksec. The spectral hardness changed in correlation with the oscillations and became significantly softer during the decline. Variations of this strength of the $\mathrm{X}$-ray flux had never been observed before in any nova in outburst. Possible mechanisms for the variations will be discussed.

1 Landessternwarte Heidelberg, Germany 
THE 2003 OUTBURST OF V4641 SGR $i=$ SAX 1819.3-2525) C. Bailyn 273 THE NATURE OF ULTRALUMINOUS SOURCES: X-RAY AND OPTICAL CLUES M. W. Pakull 273 JET INDUCED NUCLEOSYNTHESIS IN MISALIGNED MICROQUASARS Y. Butt 273 THEORETICAL MODELS OF X-RAY BINARIES IN NEARBY GALAXIES $V$. Kalogera 274 THE EXTRAORDINARY LUMINOUS BINARY STAR, QU CAR L. Hartley 274 GALACTIC POPULATIONS OF ULTRACOMPAC BINARIES K. Belczyński
TYPE IA SUPERNOVAE AND THEIR IMPLICATIONS FOR COSMOLOGY M. Livio 274

RELATIVISTIC MEASUREMENTS OF PULSARWHITE DWARF BINARIES: NEW RESULTS FROM ARECIBO D. Nice 275 MULTIWAVELENGTH OBSERVATIONS OF RX J0806.3+1527: THE SHORTEST ORBITAL PERIOD BIN $G$. Israel 275 X-RAY OBSERVATIONS OF NOVA V4743 SAGITTARIUS IN OUTBURST J. Krautter, J.-U. Ness, S. Starrfield, V. Burwitz, J. J. Drake, G $M$. Orio 